\title{
Semileptonic $B_{(s)} \rightarrow a_{1}\left(K_{1}\right) \ell^{+} \ell^{-}$decays via the light-cone sum rules with $B$-meson distribution amplitudes
}

\author{
S. Momeni* and R. Khosravi ${ }^{\dagger}$ \\ Department of Physics, Isfahan University of Technology, Isfahan 84156-83111, Iran
}

\begin{abstract}
The form factors of semileptonic $B_{(s)} \rightarrow a_{1}\left(K_{1}\right) \ell^{+} \ell^{-}, \ell=\tau, \mu, e$ transitions are investigated in the framework of the light-cone sum rules with $B$-meson distribution amplitudes, which play an important role in exclusive $B$ decays. The $B$-meson distribution amplitudes, $\varphi_{ \pm}(\omega)$ are a modeldependent form, so we consider four different parameterizations which can provide a reasonable description of $\varphi_{ \pm}(\omega)$ from QCD corrections. The branching fractions of these transitions are calculated. For a better analysis, a comparison of our results with the prediction of other models is provided.
\end{abstract}

\section{INTRODUCTION}

Inclusive and exclusive decays of $B$-meson play a perfect role in determination of fundamental parameters used in the standard model (SM) and improve our studies in understanding the dynamics of quantum chromo dynamics (QCD). Among of all $B$ decays, the semileptonic decays occupy a special place since their theoretical description is relatively simple. In this field, reliable calculations of heavy-to-light transition form factors of semileptonic $B$ decays are very important in particle physics. These form factors are also used to determine the amplitude of non-leptonic $B$ decays applied to evaluate the CKM parameters as well as to test various properties of the SM.

In the region of large momentum transfer squared, $\left(q^{2}\right)$ heavy-to-light form factors are successfully investigated via the Lattice QCD. But in small $q^{2}$, other approaches are used such as the light-cone sum rules (LCSR) [1-3]. In the usual LCSR method, the correlation function is inserted between the vacuum and light meson. As a result of this calculation, the long distance dynamics is described by light-cone distribution amplitudes (LCDA's) of light meson [4-12]. Still, there is very limited knowledge of the nonperturbative parameters determining these LCDA's. Therefore, the main uncertainty in estimating the form factors comes from the limited accuracy of the LCDA parameters.

As the direct analogue of the LCDA's of light mesons, the $B$-meson distribution amplitudes (DA's) were introduced to describe generic exclusive $B$ decays with the contribution of the hard gluon exchange [13]. Based on the local OPE and condensate expansion, the classical two-point sum rules was used for the $B$-meson DA's already in the original study [14]. The $B$-meson DA's emerge as universal nonperturbative objects in many studies of exclusive $B$-meson decays (for instance see [15]). An estimate of the inverse moment of two-particle DA, $\varphi_{+}$was also obtained by matching the factorization formula to the LCSR for $B \rightarrow \gamma \ell \nu$ [16]. The shapes of the $B$-meson DA, $\varphi_{+}$depends on the model and our knowledge of the behaviors of $\varphi_{+}$is still rather limited due to the poor understanding of nonperturbative QCD dynamics.

Using the LCSR technique and relating the $B$-meson DA's to the $B \rightarrow \pi$ form factor, a new approach was suggested in [17]. In this new LCSR, correlation function was taken between the vacuum and $B$-meson and it was expanded in terms of $B$-meson DA's near the light-cone region. Therefore, the link was established between the $B$-meson DA's and transition form factors, which provide an independent dynamical information on the $B$-meson DA's. The new LCSR has been derived for $B \rightarrow \pi, K$ and $B \rightarrow \rho, K^{*}$ form factors in the leading order including the contributions of twoand three-particle DA's in [18]. Moreover, in this reference the $B$-meson three-particle DA's have been investigated and their form have been established at small momenta of light-quark and gluon.

In this paper the heavy-to-light decays, $B_{(s)} \rightarrow a_{1}\left(K_{1}\right) \ell^{+} \ell^{-}, \ell=e, \mu, \tau$ are described by the flavor changing neutral current (FCNC) processes via $b \rightarrow d \ell^{+} \ell^{-}$transition at quark level which proceed through the electroweak penguin and box diagrams. The exclusive FCNC $B$ decays are important for development of new physics and flavor physics beyond the SM. The main purpose of this paper is to consider the form factors of the FCNC $B_{(s)} \rightarrow a_{1}\left(K_{1}\right)$ transition with LCSR approach, using the $B$-meson DA's, and comparing these form factors with those of other approaches, especially the usual LCSR. Comparing form factor results between two independent methods establish input parameters and assumptions as well as predictions of the conventional LCSR.

*e-mail: samira.momeni@phy.iut.ac.ir

†e-mail: rezakhosravi @ cc.iut.ac.ir 
It should be noted that the physical state of $K_{1}(1270)$ meson is consider as a mixture of two $\left|{ }^{3} P_{1}\right\rangle$ and $\left|{ }^{1} P_{1}\right\rangle$ states and can be parameterized in terms of a mixing angle $\theta_{K}$, as follows [19]:

$$
\left|K_{1}(1270)\right\rangle=\sin \theta_{K}\left|{ }^{3} P_{1}\right\rangle+\cos \theta_{K}\left|{ }^{1} P_{1}\right\rangle,
$$

where $\left|{ }^{3} P_{1}\right\rangle \equiv\left|K_{1 A}\right\rangle$ and $\left|{ }^{1} P_{1}\right\rangle \equiv\left|K_{1 B}\right\rangle$ with different masses and decay constants. Also $\theta_{K}$ is the mixing angle and can be determined by the experimental data. There are various approaches to estimate the mixing angle. In [20] the result $35^{\circ}<\left|\theta_{K}\right|<55^{\circ}$ was found while in [21], two possible solutions with $\left|\theta_{K}\right| \approx 33^{\circ}$ and $57^{\circ}$ were obtained.

The contents of this paper are as follows: In section II, the effective weak Hamiltonian of the $b \rightarrow d \ell^{+} \ell^{-}$transition are presented. In section III, we derive the $B_{(s)} \rightarrow a_{1}\left(K_{1}\right) \ell^{+} \ell^{-}$form factors with the LCSR method using the $B$ meson DA's. To achieve a better analysis, we consider four different parameterizations for the shapes of the $B$-meson DA's, $\varphi_{ \pm}$. The form factors of the $B_{(s)} \rightarrow a_{1}\left(K_{1}\right) \ell^{+} \ell^{-}$decays are basic parameters in studying the exclusive nonleptonic two-body decays and semileptonic decays. Our numerical analysis of the form factors as well as branching ratio values and their comparison with the prediction of other approaches is provided in section IV.

\section{THE EFFECTIVE WEAK HAMILTONIAN OF THE $b \rightarrow d \ell^{+} \ell^{-}$TRANSITION}

In the $\mathrm{SM}$, the $B_{(s)} \rightarrow a_{1}\left(K_{1}\right) \ell^{+} \ell^{-}$decay amplitude is reduced to the matrix element defined as $\left\langle a_{1}\left(K_{1}\right) \ell^{+} \ell^{-}\left|\mathcal{H}_{\mathrm{eff}}^{b \rightarrow d}\right| B_{(s)}\right\rangle$. The effective weak Hamiltonian of the $b \rightarrow d \ell^{+} \ell^{-}$transition has the following form in the SM:

$$
H_{\mathrm{eff}}^{b \rightarrow d}=-\frac{G_{F}}{\sqrt{2}}\left(V_{u b} V_{u d}^{*} \sum_{i=1}^{2} C_{i}(\mu) O_{i}^{u}(\mu)+V_{c b} V_{c d}^{*} \sum_{i=1}^{2} C_{i}(\mu) O_{i}^{c}(\mu)-V_{t b} V_{t d}^{*} \sum_{i=3}^{10} C_{i}(\mu) O_{i}(\mu)\right),
$$

where $V_{j k}$ and $C_{i}(\mu)$ are the CKM matrix elements and Wilson coefficients, respectively. The local operators are current-current operators $O_{1,2}^{u, c}$, QCD penguin operators $O_{3-6}$, magnetic penguin operators $O_{7,8}$, and semileptonic electroweak penguin operators $O_{9,10}$. The explicit expressions of these operators for $b \rightarrow d \ell^{+} \ell^{-}$transition are written as $[22]$

$$
\begin{aligned}
& O_{1}=\left(\bar{d}_{i} c_{j}\right)_{V-A},\left(\bar{c}_{j} b_{i}\right)_{V-A}, \quad O_{2}=(\bar{d} c)_{V-A}(\bar{c} b)_{V-A}, \\
& O_{3}=(\bar{d} b)_{V-A} \sum_{q}(\bar{q} q)_{V-A}, \quad O_{4}=\left(\bar{d}_{i} b_{j}\right)_{V-A} \sum_{q}\left(\bar{q}_{j} q_{i}\right)_{V-A}, \\
& O_{5}=(\bar{d} b)_{V-A} \sum_{q}(\bar{q} q)_{V+A}, \quad O_{6}=\left(\bar{d}_{i} b_{j}\right)_{V-A} \sum_{q}\left(\bar{q}_{j} q_{i}\right)_{V+A}, \\
& O_{7}=\frac{e}{8 \pi^{2}} m_{b}\left(\bar{d} \sigma^{\mu \nu}\left(1+\gamma_{5}\right) b\right) F_{\mu \nu}, O_{8}=\frac{g}{8 \pi^{2}} m_{b}\left(\bar{d}_{i} \sigma^{\mu \nu}\left(1+\gamma_{5}\right) T_{i j} b_{j}\right) G_{\mu \nu} \text {, } \\
& O_{9}=\frac{e}{8 \pi^{2}}(\bar{d} b)_{V-A}(\bar{l} l)_{V}, \quad O_{10}=\frac{e}{8 \pi^{2}}(\bar{d} b)_{V-A}(\overline{l l})_{A},
\end{aligned}
$$

where $G_{\mu \nu}$ and $F_{\mu \nu}$ are the gluon and photon field strengths, respectively; $T_{i j}$ are the generators of the $S U(3)$ color group; $i$ and $j$ denote color indices. Labels $(V \pm A)$ stand for $\gamma^{\mu}\left(1 \pm \gamma^{5}\right)$. The magnetic and electroweak penguin operators $O_{7}$, and $O_{9,10}$ are responsible for the short distance (SD) effects in the FCNC $b \rightarrow d$ transition, but the operators $O_{1-6}$ involve both SD and long distance (LD) contributions in this transition. In the naive factorization approximation, contributions of the $O_{1-6}$ operators have the same form factor dependence as $C_{9}$ which can be absorbed into an effective Wilson coefficient $C_{9}^{\text {eff }}$. Therefore, the matrix element for the $b \rightarrow d \ell^{+} \ell^{-}$transition can be written as:

$$
\begin{aligned}
\mathcal{M} & =\frac{G_{F} \alpha}{2 \sqrt{2} \pi} V_{t b} V_{t d}^{*}\left[C_{9}^{\mathrm{eff}} \bar{d} \gamma_{\mu}\left(1-\gamma_{5}\right) b \bar{l} \gamma_{\mu} l+C_{10} \bar{d} \gamma_{\mu}\left(1-\gamma_{5}\right) b \bar{l} \gamma_{\mu} \gamma_{5} l\right. \\
& \left.-2 C_{7}^{\mathrm{eff}} \frac{m_{b}}{q^{2}} \bar{d} i \sigma_{\mu \nu} q^{\nu}\left(1+\gamma_{5}\right) b \bar{l} \gamma_{\mu} l\right]
\end{aligned}
$$

where $\bar{d} \gamma_{\mu}\left(1-\gamma_{5}\right) b$ and $\bar{d} i \sigma_{\mu \nu} q^{\nu}\left(1+\gamma_{5}\right) b$ are the transition currents denoted with $J_{\mu}^{V-A}$ and $J_{\mu}^{T}$ respectively, in this work. Eq. (4) also contains two effective Wilson coefficients $C_{7}^{\text {eff }}$ and $C_{9}^{\text {eff }}$, where $C_{7}^{\text {eff }}=C_{7}-C_{5} / 3-C_{6}$. The effective Wilson coefficient $C_{9}^{\text {eff }}$ includes both the SD and LD effects as

$$
C_{9}^{\mathrm{eff}}=C_{9}+Y_{S D}\left(q^{2}\right)+Y_{L D}\left(q^{2}\right)
$$

where $Y_{S D}\left(q^{2}\right)$ describes the SD contributions from four-quark operators far away from the resonance regions, which can be calculated reliably in perturbative theory as [22, 23]:

$$
\begin{aligned}
Y_{S D}\left(q^{2}\right) & =0.138 \omega(s)+h\left(\hat{m}_{c}, s\right) C_{0}-\frac{1}{2} h(1, s)\left(4 C_{3}+4 C_{4}+3 C_{5}+C_{6}\right) \\
& -\frac{1}{2} h(0, s)\left(2 \lambda_{u}\left[3 C_{1}+C_{2}\right]+C_{3}+4 C_{4}\right)+\frac{2}{9}\left(3 C_{3}+C_{4}+3 C_{5}+C_{6}\right),
\end{aligned}
$$


where $s=q^{2} / m_{b}^{2}, \hat{m}_{c}=m_{c} / m_{b}, C_{0}=-\lambda_{c}\left(3 C_{1}+C_{2}\right)+3 C_{3}+C_{4}+3 C_{5}+C_{6}, \lambda_{c}=\frac{V_{c b} V_{c d}^{*}}{V_{t b} V_{t d}^{*}}, \lambda_{u}=\frac{V_{u b} V_{u d}^{*}}{V_{t b} V_{t d}^{*}}$, and

$$
\begin{aligned}
\omega(s) & =-\frac{2}{9} \pi^{2}-\frac{4}{3} \mathrm{Li}_{2}(s)-\frac{2}{3} \ln (s) \ln (1-s)-\frac{5+4 s}{3(1+2 s)} \ln (1-s)-\frac{2 s(1+s)(1-2 s)}{3(1-s)^{2}(1+2 s)} \ln (s) \\
& +\frac{5+9 s-6 s^{2}}{3(1-s)(1+2 s)}
\end{aligned}
$$

represents the $\mathcal{O}\left(\alpha_{s}\right)$ correction coming from one gluon exchange in the matrix element of the operator $O_{9}$ [24], while $h\left(\hat{m}_{c}, s\right)$ and $h(0, s)$ represent one-loop corrections to the four-quark operators $O_{1-6}$ [25]. The functional form of the $h\left(\hat{m}_{c}, s\right)$ and $h(0, s)$ are as:

$$
\begin{aligned}
h\left(\hat{m}_{c}, s\right) & =-\frac{8}{9} \ln \frac{m_{b}}{\mu}-\frac{8}{9} \ln \hat{m}_{c}+\frac{8}{27}+\frac{4}{9} x \\
& -\frac{2}{9}(2+x)|1-x|^{1 / 2} \begin{cases}\left(\ln \left|\frac{\sqrt{1-x}+1}{\sqrt{1-x}-1}\right|-i \pi\right), & \text { for } x \equiv \frac{4 \hat{m}_{c}^{2}}{s}<1 \\
2 \arctan \frac{1}{\sqrt{x-1}}, & \text { for } x \equiv \frac{4 \hat{m}_{c}^{2}}{s}>1, \\
h(0, s) & =\frac{8}{27}-\frac{8}{9} \ln \frac{m_{b}}{\mu}-\frac{4}{9} \ln s+\frac{4}{9} i \pi .\end{cases}
\end{aligned}
$$

The LD contributions, $Y_{L D}\left(q^{2}\right)$ from four-quark operators near the $u \bar{u}, d \bar{d}$ and $c \bar{c}$ resonances can not be calculated from the first principles of QCD and are usually parameterized in the form of a phenomenological Breit-Wigner formula as $[22,23]$ :

$$
Y_{L D}\left(q^{2}\right)=\frac{3 \pi}{\alpha^{2}}\left\{\sum_{V_{i}=\psi(1 s), \psi(2 s)} \frac{\Gamma\left(V_{i} \rightarrow l^{+} l^{-}\right) m_{V_{i}}}{m_{V_{i}}^{2}-q^{2}-i m_{V_{i}} \Gamma_{V_{i}}}-\lambda_{u} h(0, s)\left(3 C_{1}+C_{2}\right) \sum_{V_{i}=\rho, \omega} \frac{\Gamma\left(V_{i} \rightarrow l^{+} l^{-}\right) m_{V_{i}}}{m_{V_{i}}^{2}-q^{2}-i m_{V_{i}} \Gamma_{V_{i}}}\right\} .
$$

\section{III. $B_{(s)} \rightarrow a_{1}\left(K_{1}\right) \ell^{+} \ell^{-}$FORM FACTORS WITH THE LCSR}

First, we start with the two-point correlation function to compute the form factors of the $B \rightarrow a_{1} \ell^{+} \ell^{-}$via the LCSR and then explain how to extract the $B \rightarrow K_{1}$ transition form factors. The correlation function is constructed from the transition currents $J_{\nu}^{V-A}$ and $J_{\nu}^{T}$ as follows:

$$
\Pi_{\mu \nu}^{V-A(T)}(p, q)=i \int d^{4} x e^{i p . x}\left\langle 0\left|\mathcal{T}\left\{J_{\mu}^{a_{1}}(x) J_{\nu}^{V-A(T)}(0)\right\}\right| B(P)\right\rangle .
$$

In this definition for the correlation function, $\mathcal{T}$ is the time ordering operator, $|0\rangle$ is an appropriate ground state (usually vacuum), $J_{\mu}^{a_{1}}=\bar{u} \gamma_{\mu} \gamma_{5} d$ is the interpolating current of the axial-vector meson $a_{1}$. The external momenta of the interpolating and transition currents, $J_{\mu}^{a_{1}}$ and $J_{\nu}^{V-A(T)}$, are $p$ and $q$, respectively, and $P^{2}=(p+q)^{2}=m_{B}^{2}$. The leading-order diagram for $B \rightarrow a_{1} \ell^{+} \ell^{-}$decays is depicted in Fig. 1.

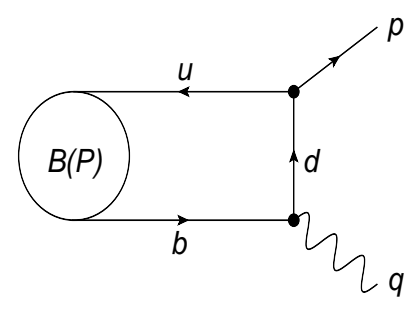

FIG. 1: leading-order diagram for $B \rightarrow a_{1} \ell^{+} \ell^{-}$decays.

According to the general philosophy of the QCD sum rules and its extension, (light-cone sum rules), the above correlation function should be calculated in two different ways. In phenomenological or physical representation, it 
is calculated in terms of hadronic parameters. In QCD side, it is obtained in terms of DA's and QCD degrees of freedom. The LCSR for the physical quantities like form factors are acquired equating coefficient of the sufficient structures from both representations of the same correlation function through the dispersion relation and applying Borel transformation and continuum subtraction to suppress the contributions of the higher states and continuum.

To obtain the phenomenological representation of the correlation function, a complete set of intermediate states with the same quantum number as the current $J_{\mu}^{a_{1}}$ is inserted in Eq. (10). Isolating the pole term of the lowest axial vector $a_{1}$ meson and applying Fourier transformation, we get

$$
\Pi_{\mu \nu}^{V-A(T)}(p, q)=\frac{1}{p^{2}-m_{a_{1}}^{2}}\left\langle 0\left|J_{\mu}^{a_{1}}(p)\right| a_{1}(p)\right\rangle\left\langle a_{1}(p)\left|J_{\nu}^{V-A(T)}\right| B(P)\right\rangle+\text { higher states . }
$$

The matrix element, $\left\langle 0\left|J_{\mu}^{a_{1}}(p)\right| a_{1}(p)\right\rangle$ is defined as

$$
\left\langle 0\left|J_{\mu}^{a_{1}}(0)\right| a_{1}(p)\right\rangle=f_{a_{1}} m_{a_{1}} \varepsilon_{\mu}
$$

where $f_{a_{1}}$ and $\varepsilon_{\mu}$ are the leptonic decay constant and polarization vector of the axial vector meson $a_{1}$, respectively. The transition matrix element, $\left\langle a_{1}(p)\left|J_{\nu}^{V-A(T)}\right| B(P)\right\rangle$, can be parameterized via Lorentz invariance and parity considerations as [26]:

$$
\begin{aligned}
\left\langle a_{1}(p)\left|J_{\mu}^{V-A}\right| B(P)\right\rangle & =\epsilon_{\mu \nu \alpha \beta} \varepsilon^{* \nu} P^{\alpha} p^{\beta} \frac{2 A\left(q^{2}\right)}{m_{B}-m_{a_{1}}}-i \varepsilon_{\mu}^{*}\left(m_{B}-m_{a_{1}}\right) V_{1}\left(q^{2}\right) \\
& +i \frac{\varepsilon^{*} \cdot P}{m_{B}-m_{a_{1}}}(P+p)_{\mu} V_{2}\left(q^{2}\right)+2 i m_{a_{1}} \frac{\varepsilon^{*} \cdot P}{q^{2}} q_{\mu}\left[V_{3}\left(q^{2}\right)-V_{0}\left(q^{2}\right)\right], \\
\left\langle a_{1}(p)\left|J_{\mu}^{T}\right| B(P)\right\rangle & =2 i \epsilon_{\mu \nu \alpha \beta} \varepsilon^{* \nu} P^{\alpha} p^{\beta} T_{1}\left(q^{2}\right)+\left[\varepsilon_{\mu}^{*}\left(m_{B}^{2}-m_{a_{1}}^{2}\right)-\left(\varepsilon^{*} \cdot P\right)(P+p)_{\mu}\right] T_{2}\left(q^{2}\right) \\
& +\left(\varepsilon^{*} \cdot P\right)\left[q_{\mu}-\frac{q^{2}}{m_{B}^{2}-m_{a_{1}}^{2}}(P+p)_{\mu}\right] T_{3}\left(q^{2}\right),
\end{aligned}
$$

$q^{2}$ is the momentum transfer squared of the $Z$ boson (photon). It should be noted that $V_{0}(0)=V_{3}(0)$ and the identity $\sigma_{\mu \nu} \gamma_{5}=\frac{-i}{2} \epsilon_{\mu \nu \alpha \beta} \sigma^{\alpha \beta}\left(\epsilon_{0123}=1\right)$ implies that $T_{1}(0)=T_{2}(0)[26]$. Moreover, $V_{3}$ can be written as a linear combination of $V_{1}$ and $V_{2}$ :

$$
V_{3}\left(q^{2}\right)=\frac{m_{B}-m_{a_{1}}}{2 m_{a_{1}}} V_{1}\left(q^{2}\right)-\frac{m_{B}+m_{a_{1}}}{2 m_{a_{1}}} V_{2}\left(q^{2}\right) .
$$

Using Eqs. (12) and (13) in Eq. (11), and performing summation over the polarization of $a_{1}$ meson, we obtain

$$
\begin{aligned}
\Pi_{\mu \nu}^{V-A} & =\frac{f_{a_{1}} m_{a_{1}}}{p^{2}-m_{a_{1}}^{2}} \times\left[\frac{2 A}{m_{B}-m_{a_{1}}}\left(q^{2}\right) \epsilon_{\mu \nu \alpha \beta} P^{\alpha} p^{\beta}-i V_{1}\left(q^{2}\right)\left(m_{B}-m_{a_{1}}\right) g_{\mu \nu}\right. \\
& \left.+i \frac{V_{2}\left(q^{2}\right)}{m_{B}-m_{a_{1}}}(P+p)_{\mu} P_{\nu}-i \frac{2 m_{a_{1}} V_{0}\left(q^{2}\right)}{q^{2}} q_{\mu} P_{\nu}\right]+ \text { higher states }, \\
\Pi_{\mu \nu}^{T} & =\frac{f_{a_{1}} m_{a_{1}}}{p^{2}-m_{a_{1}}^{2}} \times\left[2 T_{1}\left(q^{2}\right) \epsilon_{\mu \nu \alpha \beta} P^{\alpha} p^{\beta}-i T_{2}\left(q^{2}\right)\left(m_{B}^{2}-m_{a_{1}}^{2}\right) g_{\mu \nu}\right. \\
& \left.-i T_{3}\left(q^{2}\right) q_{\mu} P_{\nu}\right]+ \text { higher states. }
\end{aligned}
$$

To calculate the form factors $A, V_{i}(i=0,1,2)$ and $T_{j}(j=1,2,3)$, we will choose the structures $\epsilon_{\mu \nu \alpha \beta} P^{\alpha} p^{\beta}, g_{\mu \nu}$, $(P+p)_{\mu} P_{\nu}, q_{\mu} P_{\nu}$, from $\Pi_{\mu \nu}^{V-A}$ and $\epsilon_{\mu \nu \alpha \beta} P^{\alpha} p^{\beta}, g_{\mu \nu}$, and $q_{\mu} P_{\nu}$ from $\Pi_{\mu \nu}^{T}$, respectively. For simplicity, the correlations are written as

$$
\begin{aligned}
\Pi_{\mu \nu}^{V-A}(p, q) & =\Pi_{1} g_{\mu \nu}+\Pi_{2} \epsilon_{\mu \nu \alpha \beta} P^{\alpha} p^{\beta}+\Pi_{3}(P+p)_{\mu} P_{\nu}+\Pi_{4} q_{\mu} P_{\nu}+\ldots \ldots \\
\Pi_{\mu \nu}^{T}(p, q) & =\Pi_{1}^{\prime} g_{\mu \nu}+\Pi_{2}^{\prime} \epsilon_{\mu \nu \alpha \beta} P^{\alpha} p^{\beta}+\Pi_{3}^{\prime} q_{\mu} P_{\nu}+\ldots
\end{aligned}
$$

Now, we consider the QCD part of the correlation functions in Eq. (10) based on light-cone OPE in the heavy quark effective theory (HQET). After the transition to HQET, the correlation functions are written as [18]:

$$
\Pi_{\mu \nu}^{V-A(T)}(p, q)=\widetilde{\Pi}_{\mu \nu}^{V-A(T)}(p, \widetilde{q})+\mathcal{O}\left(1 / m_{b}\right)
$$


where $\widetilde{q}=q-m_{b} v$, and $v$ is the four-velocity of $B$-meson. Also up to $1 / m_{b}$ corrections in HQET, the state of $B$-meson $|B(P)\rangle$, and the $b$-quark field $b(x)$ are substituted by the state $|B(v)\rangle$ and the effective field $e^{-i m_{b} v x} h_{v}(x)$, respectively. Therefore, the correlation functions in the heavy quark limit, $\left(m_{b} \rightarrow \infty\right)$, become:

$$
\begin{aligned}
\widetilde{\Pi}_{\mu \nu}^{V-A}(p, \widetilde{q}) & =i \int d^{4} x e^{i p \cdot x}\left\langle 0\left|\mathcal{T}\left\{\bar{u}(x) \gamma_{\mu} \gamma_{5} i S_{d}(x) \gamma_{\nu}\left(1-\gamma_{5}\right) h_{v}(0)\right\}\right| B(v)\right\rangle \\
\widetilde{\Pi}_{\mu \nu}^{T}(p, \widetilde{q}) & =i \int d^{4} x e^{i p . x}\left\langle 0\left|\mathcal{T}\left\{\bar{u}(x) \gamma_{\mu} \gamma_{5} i S_{d}(x) \sigma_{\nu \eta} q^{\eta}\left(1+\gamma_{5}\right) h_{v}(0)\right\}\right| B(v)\right\rangle .
\end{aligned}
$$

From Eq. (18) a convolution of a short-distance part with the matrix element of the bilocal operator is obtained between the vacuum and $B(v)$-state as:

$$
\begin{aligned}
\widetilde{\Pi}_{\mu \nu}^{V-A}(p, \widetilde{q}) & =i \int d^{4} x e^{i p . x} \times\left\{\gamma_{\mu} \gamma_{5} i S_{d}(x) \gamma_{\nu}\left(1-\gamma_{5}\right)\right\}_{\alpha \beta}\left\langle 0\left|\bar{u}_{\alpha}(x) h_{v \beta}(0)\right| B(v)\right\rangle \\
\widetilde{\Pi}_{\mu \nu}^{T}(p, \widetilde{q}) & =i \int d^{4} x e^{i p . x} \times\left\{\gamma_{\mu} \gamma_{5} i S_{d}(x) \sigma_{\nu \eta} q^{\eta}\left(1+\gamma_{5}\right)\right\}_{\alpha \beta}\left\langle 0\left|\bar{u}_{\alpha}(x) h_{v \beta}(0)\right| B(v)\right\rangle .
\end{aligned}
$$

The full-quark propagator, $S_{d}(x)$ of a massless quark in the external gluon field in the Fock-Schwinger gauge is as follows:

$$
S_{d}(x)=i \int \frac{d^{4} k}{(2 \pi)^{4}} e^{-i k \cdot x}\left\{\frac{\not k}{k^{2}}-\int_{0}^{1} d v G_{\mu \nu}(v x)\left[\frac{1}{2 k^{4}} \not k \sigma^{\mu \nu}-\frac{1}{k^{2}} v x^{\mu} \gamma^{\nu}\right]\right\} .
$$

In addition, the DA's of the B-meson are as [27]:

$$
\begin{aligned}
\left\langle 0\left|\bar{u}_{\alpha}(x) h_{v \beta}(0)\right| B(v)\right\rangle= & -\frac{i f_{B} m_{B}}{4} \int_{0}^{\infty} d \omega e^{-i \omega v \cdot x}\left\{(1+\not \varphi) \varphi_{+}-\frac{\not \gamma_{5}}{2 v x}\left(\varphi_{+}-\varphi_{-}\right)\right\}_{\beta \alpha}, \\
\left\langle 0\left|\bar{u}_{\alpha}(x) G_{\lambda \rho}(u x) h_{v \beta}(0)\right| B(v)\right\rangle= & \frac{f_{B} m_{B}}{4} \int_{0}^{\infty} d \omega \int_{0}^{\infty} d \xi e^{-i(\omega+u \xi) v \cdot x}\left\{\left[\left(v_{\lambda} \gamma_{\rho}-v_{\rho} \gamma_{\lambda}\right)\left(\Psi_{A}-\Psi_{V}\right)\right.\right. \\
& \left.\left.-i \sigma_{\lambda \rho} \Psi_{V}-\frac{x_{\lambda} v_{\rho}-x_{\rho} v_{\lambda}}{v x} X_{A}+\frac{x_{\lambda} \gamma_{\rho}-x_{\rho} \gamma_{\lambda}}{v x} Y_{A}\right](1+\not \varphi) \gamma_{5}\right\}_{\beta \alpha},
\end{aligned}
$$

where [18]:

$$
\begin{aligned}
\Psi_{A}(\omega, \xi) & =\Psi_{V}(\omega, \xi)=\frac{\lambda_{E}^{2}}{6 \omega_{0}^{4}} \xi^{2} e^{-\frac{\omega+\xi}{\omega_{0}}} \\
X_{A}(\omega, \xi) & =\frac{\lambda_{E}^{2}}{6 \omega_{0}^{4}} \xi(2 \omega-\xi) e^{-\frac{\omega+\xi}{\omega_{0}}} \\
Y_{A}(\omega, \xi) & =-\frac{\lambda_{E}^{2}}{24 \omega_{0}^{4}} \xi\left(7 \omega_{0}-13 \omega+3 \xi\right) e^{-\frac{\omega+\xi}{\omega_{0}}} .
\end{aligned}
$$

Our knowledge of the behaviors of $\varphi_{ \pm}(\omega)$ at small $\omega$ is still rather limited due to the poor understanding of nonperturbative QCD dynamics. To achieve a better understanding of the model dependence of $\varphi_{ \pm}(\omega)$ in the sum rule analysis, we consider the following four different parameterizations for the shapes of the $B$-meson DA $\varphi_{+}[14,28-30]$ :

$$
\begin{aligned}
\varphi_{+, \mathrm{I}}(\omega) & =\frac{\omega}{\omega_{0}^{2}} e^{-\omega / \omega_{0}}, \\
\varphi_{+, \mathrm{II}}(\omega) & =\frac{1}{4 \pi \omega_{0}} \frac{k}{k^{2}+1}\left[\frac{1}{k^{2}+1}-\frac{2\left(\sigma_{B}-1\right)}{\pi^{2}} \ln k\right], \quad k=\frac{\omega}{1 \mathrm{GeV}}, \\
\varphi_{+, \mathrm{III}}(\omega) & =\frac{2 \omega^{2}}{\omega_{0} \omega_{1}^{2}} e^{-\left(\omega / \omega_{1}\right)^{2}}, \quad \omega_{1}=\frac{2 \omega_{0}}{\sqrt{\pi}}, \quad \omega_{2}=\frac{4 \omega_{0}}{4-\pi} . \\
\varphi_{+, \mathrm{IV}}(\omega) & =\frac{\omega}{\omega_{0} \omega_{2}} \frac{\omega_{2}-\omega}{\sqrt{\omega\left(2 \omega_{2}-\omega\right)}} \theta\left(\omega_{2}-\omega\right), \quad
\end{aligned}
$$

The determination of coefficient $\omega_{0}$, which constitutes the most important theory uncertainty in the $B$-meson LCSR approach, will be discussed for each of the four models in the next section. 
The corresponding expression of $\varphi_{-}(\omega)$ for each model is determined by:

$$
\varphi_{-}(\omega)=\int_{0}^{1} \frac{d \xi}{\xi} \varphi_{+}(\omega / \xi) .
$$

These parameterizations can provide a reasonable description of $\varphi_{ \pm}(\omega)$ at small $\omega$ due to the radiative tail developed from QCD corrections.

Inserting the full propagator and B-meson DA's presented in Eqs. (20) and (21), respectively, in the correlation functions (Eq. (19)), traces and then integrals should be calculated. To estimate these calculations, we have used $x_{\mu} \rightarrow i \frac{\partial}{\partial k_{\mu}}$. In addition to this, for terms containing a factor of $v x$ in the denominator, we have used the following trick: in order not to have any singularity at $v \cdot x=0$, the integral of these wave functions in the absence of the exponential should cancel. Hence, for these terms only, one can write:

$$
e^{i \alpha v \cdot x} \rightarrow e^{i \alpha v \cdot x}-1=i v \cdot x \int_{0}^{\alpha} d k e^{i k v \cdot x}
$$

and the rest of the calculation is similar to the presented one. Note that the subtracted 1 does not contribute.

After completing the integrals and matching them with the hadronic representation below the continuum threshold $s_{0}$, through the dispersion relation and applying Borel transform with respect to the variable $p^{2}$ as:

$$
B_{p^{2}}\left(M^{2}\right)\left(\frac{1}{p^{2}-m^{2}}\right)^{n}=\frac{(-1)^{n}}{\Gamma(n)} \frac{e^{-\frac{m^{2}}{M^{2}}}}{\left(M^{2}\right)^{n}},
$$

in order to suppress the contributions of the higher states, the form factors are obtained via the LCSR. For instance, the form factor $V_{1}$ is presented here:

$$
\begin{aligned}
V_{1}\left(q^{2}\right) & =-\frac{f_{B} m_{B}}{f_{a} m_{a_{1}}\left(m_{B}-m_{a_{1}}\right)} e^{\frac{m_{a_{1}}^{2}}{M^{2}}}\left\{\int_{0}^{\sigma_{0}} d \sigma\left[\frac{M^{2}}{2} \varphi_{+}\left(\omega^{\prime}\right) \frac{d}{d \sigma} e^{-\frac{s}{M^{2}}}\right]+\hat{\mathcal{L}}\left[(1-u) \Psi_{V} \frac{d}{d \sigma} e^{-\frac{s}{M^{2}}}\right.\right. \\
& -\frac{2 u-1}{2 M^{2}}\left(\Psi_{A}-\Psi_{V}\right) \frac{d}{d \sigma} e^{-\frac{s}{M^{2}}}+\frac{m_{B}^{2}(1-2 u)}{\bar{\sigma}^{2} M^{2}}\left(3 \widetilde{X}_{A}-\widetilde{Y}_{A}\right) e^{-\frac{s}{M^{2}}}+\frac{1}{\bar{\sigma}^{3} M^{2}} \tilde{X}_{A} e^{-\frac{s}{M^{2}}} \\
& \left.\left.\times\left(m_{B}^{2}(1+3 \sigma)-2 \widetilde{m}_{B}^{2}+\frac{\mathcal{M}^{4}}{2 M^{2}}\right)\right]\right\}
\end{aligned}
$$

The explicit expressions for the other form factors are presented in Appendix.

Finally, with a little bit of change in the previous steps, such as the change in the quark spectator $(u \rightarrow s)$, we can easily find similar results for the form factors of the $B_{s} \rightarrow K_{1 A}$, and $B_{s} \rightarrow K_{1 B}$ decays.

The form factors of $B_{s} \rightarrow K_{1}(1270) \ell^{+} \ell^{-}$transitions with the mixing angle $\theta_{K}$ are defined as [31]

$$
f^{K_{1}(1270)}=C_{1} \sin \theta_{K} f^{K_{1 A}}+C_{2} \cos \theta_{K} f^{K_{1 B}},
$$

where $f^{K_{1}(1270)}, f^{K_{1 A}}$ and $f^{K_{1 B}}$ stand for the form factors $A, V_{i}(i=0,1,2), T_{j}(j=1,2,3)$ of $B_{s} \rightarrow K_{1}(1270), B_{s} \rightarrow$ $K_{1 A}$ and $B_{s} \rightarrow K_{1 B}$ decays, respectively. The coefficients $C_{1}$ and $C_{2}$ related to each form factor of $B_{s} \rightarrow K_{1}(1270)$ decay are given in Table I.

TABLE I: The coefficients $C_{1}$ and $C_{2}$ for each form factor of $B_{s} \rightarrow K_{1}(1270)$.

\begin{tabular}{ccc}
\hline \hline Form factors & $C_{1}$ & $C_{2}$ \\
\hline$A, V_{1}, V_{2}$ & $\frac{m_{B_{s}}-m_{K_{1}}}{m_{B_{s}}-m_{K_{1 A}}}$ & $\frac{m_{B_{s}}-m_{K_{1}}}{m_{B_{s}}-m_{K_{1 B}}}$ \\
$V_{0}$ & $\frac{m_{K_{1 A}}}{m_{K_{1}}}$ & $\frac{m_{K_{1 B}}}{m_{K_{1}}}$ \\
$T_{1}, T_{3}$ & 1 & 1 \\
$T_{2}$ & $\frac{m_{B_{s}}^{2}-m_{K_{1 A}}^{2}}{m_{B_{s}}^{2}-m_{K_{1}}^{2}}$ & $\frac{m_{B_{s}}^{2}-m_{K_{1 B}}^{2}}{m_{B_{s}}^{2}-m_{K_{1}}^{2}}$ \\
\hline \hline
\end{tabular}




\section{NUMERICAL ANALYSIS}

In this section, our numerical analysis of the form factors $A, V_{i}$ and $T_{j}$ are presented for the $B_{(s)} \rightarrow a_{1}\left(K_{1}\right) \ell^{+} \ell^{-}$ decays. The values are chosen for masses in $\mathrm{GeV}$ as $m_{B}=(5.27 \pm 0.01), m_{a_{1}}=(1.23 \pm 0.04), m_{K_{1}}=(1.27 \pm 0.01)$, $m_{\mu}=0.11$ and $m_{\tau}=1.77$ [32], $m_{K_{1 A}}=(1.31 \pm 0.06), m_{K_{1 B}}=(1.34 \pm 0.08)$ [33]. The leptonic decay constants are taken as: $f_{a_{1}}=(0.24 \pm 0.01) \mathrm{GeV}, f_{K_{1 A}}=(0.25 \pm 0.01) \mathrm{GeV}, f_{K_{1 B}}=(0.19 \pm 0.01) \mathrm{GeV}[33], f_{B}=(0.18 \pm 0.02) \mathrm{GeV}$ [34], and $f_{B_{s}}=(0.23 \pm 0.03) \mathrm{GeV}$ [35]. Moreover, $s_{0}=(2.55 \pm 0.15) \mathrm{GeV}^{2}$ is used for the continuum threshold [33] . The values of the parameters $\lambda_{E}^{2}$ and $\sigma_{B}$ of the $B$-meson DA's are chosen as $\lambda_{E}^{2}=(0.11 \pm 0.06) \mathrm{GeV}^{2}[14]$ and $\sigma_{B}=1.4 \pm 0.4$ [28]. The Borel parameter in this article is taken as $1.5 \mathrm{GeV}^{2} \leq \mathrm{M}^{2} \leq 4 \mathrm{GeV}^{2}$. In this region, the values of the form factors $A, V_{i}$ and $T_{j}$ are stable enough. The uncertainties which originated from the Borel parameter $M^{2}$ in this interval, are about $1 \%$.

Having all these input values and parameters at hand, we proceed to carry out numerical calculations. As can be seen in Eq. (23), the $B$-meson DA's, $\varphi_{ \pm}$in the four cases are related to the parameter $\omega_{0}$ whose value is depend on the model. In order to determine the parameter $\omega_{0}$ for $B \rightarrow a_{1} \ell^{+} \ell^{-}$decay, we match the values of the form factor $A^{B \rightarrow a_{1}}$ in $q^{2}=0$, estimated with the four models of the $B$-meson DA's $\varphi_{ \pm}$, with $A^{B \rightarrow a_{1}}(0)=0.26 \pm 0.09$ computed from the PQCD as a different method [35], and derive the values of the coefficient $\omega_{0}$ for each model. Also, taking $A^{B_{s} \rightarrow K_{1 A}}(0)=0.25 \pm 0.10$ and $A^{B_{s} \rightarrow K_{1 B}}(0)=0.18 \pm 0.08$ evaluated via the PQCD [35], and performing the same procedure as $B \rightarrow a_{1}$ decay for $B_{s} \rightarrow K_{1 A}$ and $B_{s} \rightarrow K_{1 B}$ transitions, the values of the parameter $\omega_{0}$ are calculated for these decays. The values of the parameter $\omega_{0}$ for three aforementioned decays are given in Table II. Fig. 2 shows

TABLE II: The values of $\omega_{0}$ for each model in $\mathrm{MeV}$.

\begin{tabular}{|c|c|c|c|c|}
\hline Model & I & II & III & IV \\
\hline$\omega_{0}\left(\right.$ for $\left.B \rightarrow a_{1}\right)$ & $235_{-19}^{+25}$ & $246_{-21}^{+28}$ & $259_{-22}^{+29}$ & $\overline{217_{-16}^{+20}}$ \\
\hline$\omega_{0}\left(\right.$ for $\left.B_{s} \rightarrow K_{1 A}\right)$ & $254_{-20}^{+27}$ & $267_{-22}^{+29}$ & $281_{-25}^{+33}$ & $234_{-17}^{+23}$ \\
\hline$\omega_{0}\left(\right.$ for $\left.B_{s} \rightarrow K_{1 B}\right)$ & $282_{-22}^{+33}$ & $298_{-26}^{+34}$ & $313_{-28}^{+37}$ & $259_{-20}^{+23}$ \\
\hline
\end{tabular}

the form factors $A^{B \rightarrow a_{1}}, A^{B_{s} \rightarrow K_{1 A}}$ and $A^{B_{s} \rightarrow K_{1 B}}$ with the four models of the $B$-meson DA's $\varphi_{ \pm}$whose values at zero momentum transfer have been fixed to the predictions from the PQCD. In this figure, blue lines show the form factors predicted by the PQCD method.
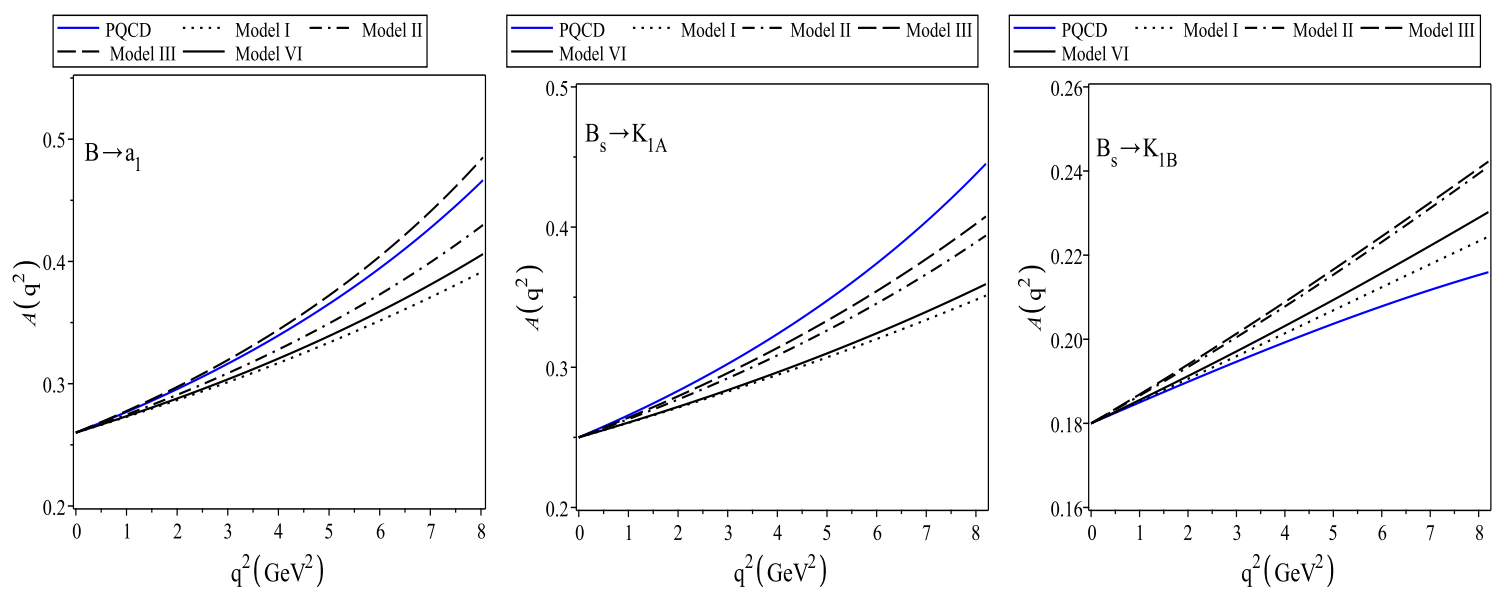

FIG. 2: Dotted, dot-dashed, dashed, solid (black) curves show the form factors $A$, calculated with the $B$-meson DA's, whose values at $q^{2}=0$ have been fixed to the prediction from the PQCD (blue).

Now, by inserting the values of the masses, leptonic decay constants, continuum threshold, Borel mass, the parameters of the $B$-meson DA's such as $\omega_{0}$ and other quantities that appear in the form factors, we can calculate the form factors of $B_{(s)} \rightarrow a_{1}\left(K_{1 A}, K_{1 B}\right)$ decays at zero momentum transfer. Taking into account all the uncertainties, the numerical values of the form factors $A, V_{i}$ and $T_{j}$ for aforementioned decays in $q^{2}=0$ are presented in Table III for the four models of the $B$-meson DA's, $\varphi_{ \pm}$. The main uncertainty comes from $\omega_{0}$, the decay constant $f_{a_{1}}\left(f_{K_{1 A}}, f_{K_{1 B}}\right)$, and $B$-meson mass.

So far, several authors have calculated the form factors of the $B_{(s)} \rightarrow a_{1}\left(K_{1 A}, K_{1 B}\right) \ell^{+} \ell^{-}$decays via differen 
TABLE III: The $B_{(s)} \rightarrow a_{1}\left(K_{1 A}, K_{1 B}\right)$ form factors at zero momentum transfer in the four models of $B$-meson DA's, $\varphi_{ \pm}$.

\begin{tabular}{|c|c|c|c|c|c|c|}
\hline Model & $A^{B \rightarrow a_{1}}$ & $V_{1}^{B \rightarrow a_{1}}$ & $V_{2}^{B \rightarrow a_{1}}$ & $V_{0}^{B \rightarrow a_{1}}$ & $T_{1}^{B \rightarrow a_{1}}=T_{2}^{B \rightarrow a_{1}}$ & $T_{3}^{B \rightarrow a_{1}}$ \\
\hline I & $0.26 \pm 0.09$ & $0.42 \pm 0.13$ & $0.22 \pm 0.07$ & $0.11 \pm 0.03$ & $0.25 \pm 0.08$ & $0.22 \pm 0.06$ \\
\hline II & $0.26 \pm 0.09$ & $0.51 \pm 0.16$ & $0.25 \pm 0.08$ & $0.13 \pm 0.04$ & $0.30 \pm 0.09$ & $0.25 \pm 0.07$ \\
\hline III & $0.26 \pm 0.09$ & $0.54 \pm 0.17$ & $0.28 \pm 0.09$ & $0.27 \pm 0.08$ & $0.34 \pm 0.11$ & $0.29 \pm 0.09$ \\
\hline VI & $0.26 \pm 0.09$ & $0.40 \pm 0.13$ & $0.20 \pm 0.06$ & $0.11 \pm 0.03$ & $0.24 \pm 0.07$ & $0.21 \pm 0.06$ \\
\hline Model & $A^{B_{s} \rightarrow K_{1 A}}$ & $V_{1}^{B_{s} \rightarrow K_{1 A}}$ & $V_{2}^{B_{s} \rightarrow K_{1 A}}$ & $V_{0}^{B_{s} \rightarrow K_{1 A}}$ & $T_{1}^{B_{s} \rightarrow K_{1 A}}=T_{2}^{B_{s} \rightarrow K_{1 A}}$ & $T_{3}^{B_{s} \rightarrow K_{1 A}}$ \\
\hline I & $0.25 \pm 0.10$ & $0.35 \pm 0.12$ & $0.15 \pm 0.05$ & $0.12 \pm 0.04$ & $0.27 \pm 0.09$ & $0.24 \pm 0.08$ \\
\hline II & $0.25 \pm 0.10$ & $0.37 \pm 0.13$ & $0.18 \pm 0.05$ & $0.11 \pm 0.03$ & $0.32 \pm 0.11$ & $0.27 \pm 0.09$ \\
\hline III & $0.25 \pm 0.10$ & $0.45 \pm 0.15$ & $0.22 \pm 0.07$ & $0.12 \pm 0.04$ & $0.39 \pm 0.13$ & $0.33 \pm 0.12$ \\
\hline VI & $0.25 \pm 0.10$ & $0.33 \pm 0.11$ & $0.13 \pm 0.04$ & $0.11 \pm 0.03$ & $0.25 \pm 0.09$ & $0.21 \pm 0.07$ \\
\hline Model & $A^{B_{s} \rightarrow K_{1 B}}$ & $V_{1}^{B_{s} \rightarrow K_{1 B}}$ & $V_{2}^{B_{s} \rightarrow K_{1 B}}$ & $V_{0}^{B_{s} \rightarrow K_{1 B}}$ & $T_{1}^{B_{s} \rightarrow K_{1 B}}=T_{2}^{B_{s} \rightarrow K_{1 B}}$ & $T_{3}^{B_{s} \rightarrow K_{1 B}}$ \\
\hline $\mathrm{I}$ & $0.18 \pm 0.08$ & $0.28 \pm 0.10$ & $0.12 \pm 0.04$ & $0.07 \pm 0.02$ & $0.22 \pm 0.08$ & $0.19 \pm 0.07$ \\
\hline II & $0.18 \pm 0.08$ & $0.33 \pm 0.12$ & $0.15 \pm 0.05$ & $0.10 \pm 0.03$ & $0.28 \pm 0.10$ & $0.25 \pm 0.09$ \\
\hline III & $0.18 \pm 0.08$ & $0.38 \pm 0.14$ & $0.20 \pm 0.07$ & $0.12 \pm 0.03$ & $0.33 \pm 0.11$ & $0.30 \pm 0.11$ \\
\hline VI & $0.18 \pm 0.08$ & $0.27 \pm 0.08$ & $0.11 \pm 0.03$ & $0.09 \pm 0.02$ & $0.21 \pm 0.07$ & $0.18 \pm 0.05$ \\
\hline
\end{tabular}

frameworks. To compare the results, we should rescale them according to the form factor definitions in Eq. (13). Table IV shows the values of the rescaled form factors at $q^{2}=0$ from different approaches. Considering the uncertainties,

TABLE IV: Transition form factors of the $B_{(s)} \rightarrow a_{1}\left(K_{1 A}, K_{1 B}\right) \ell^{+} \ell^{-}$at $q^{2}=0$ in various methods.

\begin{tabular}{|c|c|c|c|c|c|c|}
\hline Approaches & $A^{B \rightarrow a_{1}}$ & $V_{1}^{B \rightarrow a_{1}}$ & $V_{2}^{B \rightarrow a_{1}}$ & $V_{0}^{B \rightarrow a_{1}}$ & $T_{1}^{B \rightarrow a_{1}}=T_{2}^{B \rightarrow a_{1}}$ & $T_{3}^{B \rightarrow a_{1}}$ \\
\hline PQCD [35] & $0.26 \pm 0.09$ & $0.43 \pm 0.16$ & $0.13 \pm 0.04$ & $0.34 \pm 0.16$ & $0.34 \pm 0.13$ & $0.30 \pm 0.17$ \\
\hline LCSR [36] & $0.42 \pm 0.16$ & $0.68 \pm 0.13$ & $0.31 \pm 0.16$ & $0.30 \pm 0.18$ & $0.44 \pm 0.28$ & $0.41 \pm 0.18$ \\
\hline \multirow[t]{2}{*}{ 3PSR [37] } & 0.51 & 0.52 & 0.25 & 0.76 & 0.37 & 0.41 \\
\hline & $A^{B_{s} \rightarrow K_{1 A}}$ & $V_{1}^{B_{s} \rightarrow K_{1 A}}$ & $V_{2}^{B_{s} \rightarrow K_{1 A}}$ & $V_{0}^{B_{s} \rightarrow K_{1 A}}$ & $T_{1}^{B_{s} \rightarrow K_{1 A}}=T_{2}^{B_{s} \rightarrow K_{1 A}}$ & $T_{3}^{B_{s} \rightarrow K_{1 A}}$ \\
\hline \multirow[t]{2}{*}{ PQCD [35] } & $0.25 \pm 0.10$ & $0.43 \pm 0.19$ & $0.11 \pm 0.05$ & $0.36 \pm 0.18$ & $0.34 \pm 0.15$ & $0.30 \pm 0.13$ \\
\hline & $A^{B_{s} \rightarrow K_{1 B}}$ & $V_{1}^{B_{s} \rightarrow K_{1 B}}$ & $V_{2}^{B_{s} \rightarrow K_{1 B}}$ & $V_{0}^{B_{s} \rightarrow K_{1 B}}$ & $T_{1}^{B_{s} \rightarrow K_{1 B}}=T_{2}^{B_{s} \rightarrow K_{1 B}}$ & $T_{3}^{B_{s} \rightarrow K_{1 B}}$ \\
\hline PQCD [35] & $0.18 \pm 0.08$ & $0.33 \pm 0.14$ & $0.03 \pm 0.03$ & $0.42 \pm 0.16$ & $0.26 \pm 0.11$ & $0.17 \pm 0.08$ \\
\hline
\end{tabular}

our results for the form factors of these decays are in a good agreement with those of the PQCD in most cases (except $V_{2}$ and $V_{0}$ ). However, there is not good agreement between our results with the LCSR with $a_{1}$-meson DA's [36].

The LCSR calculations for the form factors are truncated at about $0 \leq q^{2} \leq 8 \mathrm{GeV}^{2}$. To extend the $q^{2}$ dependence of the form factors to the full physical region, where the LCSR results are not valid, we find that the sum rules predictions for the form factors are well fitted to the following function:

$$
F_{i}\left(q^{2}\right)=\frac{F_{i}(0)}{1-\alpha\left(q^{2} / m_{B_{(s)}}^{2}\right)+\beta\left(q^{2} / m_{B_{(s)}}^{2}\right)^{2}},
$$

where $F_{i}(0), \alpha$ and $\beta$ are the constant fitted parameters. The values of the parameters $[\alpha, \beta]$ are presented in Tables V, VI and VII for $B \rightarrow a_{1}, B_{s} \rightarrow K_{1 A}$ and $B_{s} \rightarrow K_{1 B}$, respectively. The values of parameter $F_{i}(0)$ expressed the form factor results at $q^{2}=0$ were listed in Table III, before.

By averaging the values of the form factors derived from the four models of the $B$-meson DA's $\varphi_{ \pm}$at some points of $q^{2}$, and then extrapolating to the fit function in Eq. (29), we can investigate average form factors. The parameters $F_{i}(0), \alpha$ and $\beta$ for the average form factors of $B_{(s)} \rightarrow a_{1}\left(K_{1}\right)$ are given in Table VIII. For $B_{s} \rightarrow K_{1} \ell^{+} \ell^{-}$transition, the average form factors are calculated at $\theta_{K}=45^{\circ}$. Fig. 3 show the form factors with the four models, for instance $V_{0}\left(q^{2}\right)$ and $T_{1}\left(q^{2}\right)$ with respect to $q^{2}$, on which blue lines display the average form factors. Considering the uncertainties, the average form factors $V_{0}\left(q^{2}\right)$ and $T_{1}\left(q^{2}\right)$ of the $B_{(s)} \rightarrow a_{1}\left(K_{1}\right)$ decays with their uncertainty regions are displayed on $q^{2}$ in Fig. 4.

Now, we are ready to evaluate the branching ratio values for the $B_{(s)} \rightarrow a_{1}\left(K_{1}\right) \ell^{+} \ell^{-}$decays. The expression of double differential decay rate $d^{2} \Gamma / d q^{2} d \cos \theta_{\ell}$ for the $B_{(s)} \rightarrow a_{1}\left(K_{1}\right)$ transitions can be found in [26, 38]. This 
TABLE V: The parameters $[\alpha, \beta]$ obtained for the form factors of the $B \rightarrow a_{1}$ transition in the four models.

\begin{tabular}{cccccccc}
\hline \hline Model & $A\left(q^{2}\right)$ & $V_{1}\left(q^{2}\right)$ & $V_{2}\left(q^{2}\right)$ & $V_{0}\left(q^{2}\right)$ & $T_{1}\left(q^{2}\right)$ & $T_{2}\left(q^{2}\right)$ & $T_{3}\left(q^{2}\right)$ \\
\hline I & {$[1.33,0.57]$} & {$[0.87,0.38]$} & {$[1.01,0.53]$} & {$[1.04,0.29]$} & {$[1.11,0.21]$} & {$[1.25,0.48]$} & {$[1.13,1.10]$} \\
II & {$[1.51,0.50]$} & {$[1.15,0.36]$} & {$[1.38,0.54]$} & {$[1.28,0.26]$} & {$[1.29,0.47]$} & {$[1.23,0.29]$} & {$[1.29,0.94]$} \\
III & {$[1.70,0.64]$} & {$[1.20,0.46]$} & {$[1.28,0.73]$} & {$[1.15,0.31]$} & {$[1.36,0.52]$} & {$[1.30,0.35]$} & {$[1.62,0.87]$} \\
IV & {$[1.34,0.46]$} & {$[0.88,0.30]$} & {$[1.03,0.42]$} & {$[1.08,0.22]$} & {$[1.41,0.17]$} & {$[1.31,0.38]$} & {$[1.14,0.88]$} \\
\hline \hline
\end{tabular}

TABLE VI: The same as Table $\mathrm{V}$ but for $B_{s} \rightarrow K_{1 A}$ transition.

\begin{tabular}{|c|c|c|c|c|c|c|c|}
\hline Model & $A\left(q^{2}\right)$ & $V_{1}\left(q^{2}\right)$ & $V_{2}\left(q^{2}\right)$ & $V_{0}\left(q^{2}\right)$ & $T_{1}\left(q^{2}\right)$ & $T_{2}\left(q^{2}\right)$ & $T_{3}\left(q^{2}\right)$ \\
\hline I & {$[1.16,0.53]$} & {$[0.98,0.24]$} & {$[1.14,0.39]$} & {$[0.96,0.34]$} & {$[1.30,0.17]$} & {$[1.09,0.46]$} & {$[0.95,1.17]$} \\
\hline II & {$[1.44,0.56]$} & {$[1.31,0.32]$} & {$[1.20,0.49]$} & {$[1.25,0.34]$} & {$[1.34,0.28]$} & {$[1.33,0.35]$} & {$[1.08,0.91]$} \\
\hline III & {$[1.56,0.72]$} & {$[1.52,0.31]$} & {$[1.37,0.76]$} & {$[1.32,0.43]$} & {$[1.46,0.50]$} & {$[1.38,0.39]$} & {$[1.12,0.98]$} \\
\hline VI & {$[1.18,0.42]$} & {$[1.01,0.45]$} & {$[1.15,0.31]$} & {$[0.99,0.27]$} & {$[1.33,0.13]$} & {$[1.11,0.36]$} & {$[0.96,1.03]$} \\
\hline
\end{tabular}

expression contains the Wilson coefficients, the CKM matrix elements, the form factors related to the fit functions, series of functions and constants. The numerical values of the Wilson coefficients are taken from Ref. [39]. The corresponding values are listed in Table IX in the scale $\mu=m_{b}$. The other parameters can be found in [26]. After numerical analysis, the dependency of the differential branching ratios for $B \rightarrow a_{1} \ell^{+} \ell^{-}$on $q^{2}$ using the average form factors, with and without LD effects is shown in Fig. 5 for charged lepton case.

In Table X, we present the branching ratio values for muon and tau without and with LD effects using the form factors derived in the four models of $\varphi_{ \pm}$. We also estimate the branching ratio values with the average form factors $(\mathrm{AFF})$. For $B_{s} \rightarrow K_{1}$ transitions, we have calculated the average value of branching ratios in the region $35^{\circ}<\left|\theta_{K}\right|<$ $55^{\circ}$. Here, we should also stress that the results obtained for the electron are very close to those of the muon; and for this reason, we only present the branching ratios for muon in our table. In Ref. [36] via the LCSR with $a_{1}$-meson DA's, the branching ratio values of $B \rightarrow a_{1} \mu^{+} \mu^{-}$and $B \rightarrow a_{1} \tau^{+} \tau^{-}$decays by considering SD+ LD effects are predicted $(2.52 \pm 0.62) \times 10^{-8}$ and $(0.31 \pm 0.06) \times 10^{-9}$, respectively. Our results are in a good agreement with its prediction for tau case.

In summary, we calculated the transition form factors of the $B_{(s)} \rightarrow a_{1}\left(K_{1}\right) \ell^{+} \ell^{-}$decays via the LCSR with the $B$-meson DA's, $\varphi_{ \pm}$in four models. The main uncertainty comes from the $\omega_{0}$ as a parameter of the $B$-meson DA's. We estimated the branching ratio values for these decays. The dependence of the differential branching ratios on $q^{2}$ were investigated. The results for branching fraction of $B \rightarrow a_{1} \tau^{+} \tau^{-}$are in a good agreement with the usual LCSR method in Ref. [36]. However, there is not good agreement between our results for the form factors of $B \rightarrow a_{1}$ decays in $q^{2}=0$ with those of the LCSR method.

\section{Acknowledgments}

Partial support of the Isfahan university of technology research council is appreciated. 
TABLE VII: The same as Table $\mathrm{V}$ but for $B_{s} \rightarrow K_{1 B}$ transition.

\begin{tabular}{|c|c|c|c|c|c|c|c|}
\hline Model & $A\left(q^{2}\right)$ & $V_{1}\left(q^{2}\right)$ & $V_{2}\left(q^{2}\right)$ & $V_{0}\left(q^{2}\right)$ & $T_{1}\left(q^{2}\right)$ & $T_{2}\left(q^{2}\right)$ & $T_{3}\left(q^{2}\right)$ \\
\hline I & {$[0.83,0.48]$} & {$[0.62,0.29]$} & {$[0.75,0.49]$} & {$[0.77,0.37]$} & {$[0.96,0.31]$} & {$[0.78,0.37]$} & {$[0.63,1.47$} \\
\hline II & {$[1.03,0.50]$} & {$[0.81,0.35]$} & {$[0.81,0.41]$} & {$[0.93,0.40]$} & {$[0.99,0.28]$} & {$[0.99,0.28]$} & {$[0.71,1.14$} \\
\hline III & {$[1.08,0.63]$} & {$[0.90,0.28]$} & {$[0.80,0.77]$} & {$[0.96,0.42]$} & {$[1.01,0.56]$} & {$[0.91,0.24]$} & {$[0.38,1.04$} \\
\hline VI & {$[0.87,0.37]$} & {$[0.66,0.22]$} & {$[0.81,0.39]$} & {$[0.82,0.29]$} & {$[0.98,0.23]$} & {$[0.84,0.28]$} & {$[0.66,1.18$} \\
\hline
\end{tabular}

TABLE VIII: The parameters $F_{i}(0), \alpha$, and $\beta$ obtained for the average form factors of the $B_{(s)} \rightarrow a_{1}\left(K_{1}\right)$ transitions.

\begin{tabular}{|c|c|c|c|}
\hline Form factor & $F_{i}(0)$ & $\alpha$ & $\beta$ \\
\hline$\left[A^{B \rightarrow a_{1}}, A^{B_{s} \rightarrow K_{1}}\right]$ & {$[0.26,0.30]$} & {$[1.51,1.17]$} & {$[0.53,0.45]$} \\
\hline$\left[V_{1}^{B \rightarrow a_{1}}, V_{1}^{B_{s} \rightarrow K_{1}}\right]$ & {$[0.46,0.48]$} & {$[1.05,1.43]$} & {$[0.36,0.16]$} \\
\hline$\left[V_{2}^{B \rightarrow a_{1}}, V_{2}^{B_{s} \rightarrow K_{1}}\right]$ & {$[0.23,0.23]$} & {$[1.18,1.08]$} & {$[0.55,0.43]$} \\
\hline$\left[V_{0}^{B \rightarrow a_{1}}, V_{0}^{B_{s} \rightarrow K_{1}}\right]$ & {$[0.25,0.15]$} & {$[1.17,1.01]$} & {$[0.28,0.30]$} \\
\hline$\left[T_{1}^{B \rightarrow a_{1}}, T_{1}^{B_{s} \rightarrow K_{1}}\right]$ & {$[0.28,0.40]$} & {$[1.32,1.18]$} & {$[0.36,0.25]$} \\
\hline$\left[T_{2}^{B \rightarrow a_{1}}, T_{2}^{B_{s} \rightarrow K_{1}}\right]$ & {$[0.28,0.40]$} & {$[1.29,1.08]$} & {$[0.38,0.27]$} \\
\hline$\left[T_{3}^{B \rightarrow a_{1}}, T_{3}^{B_{s} \rightarrow K_{1}}\right]$ & {$[0.24,0.34]$} & {$[1.32,0.86]$} & {$[0.83,1.02]$} \\
\hline
\end{tabular}
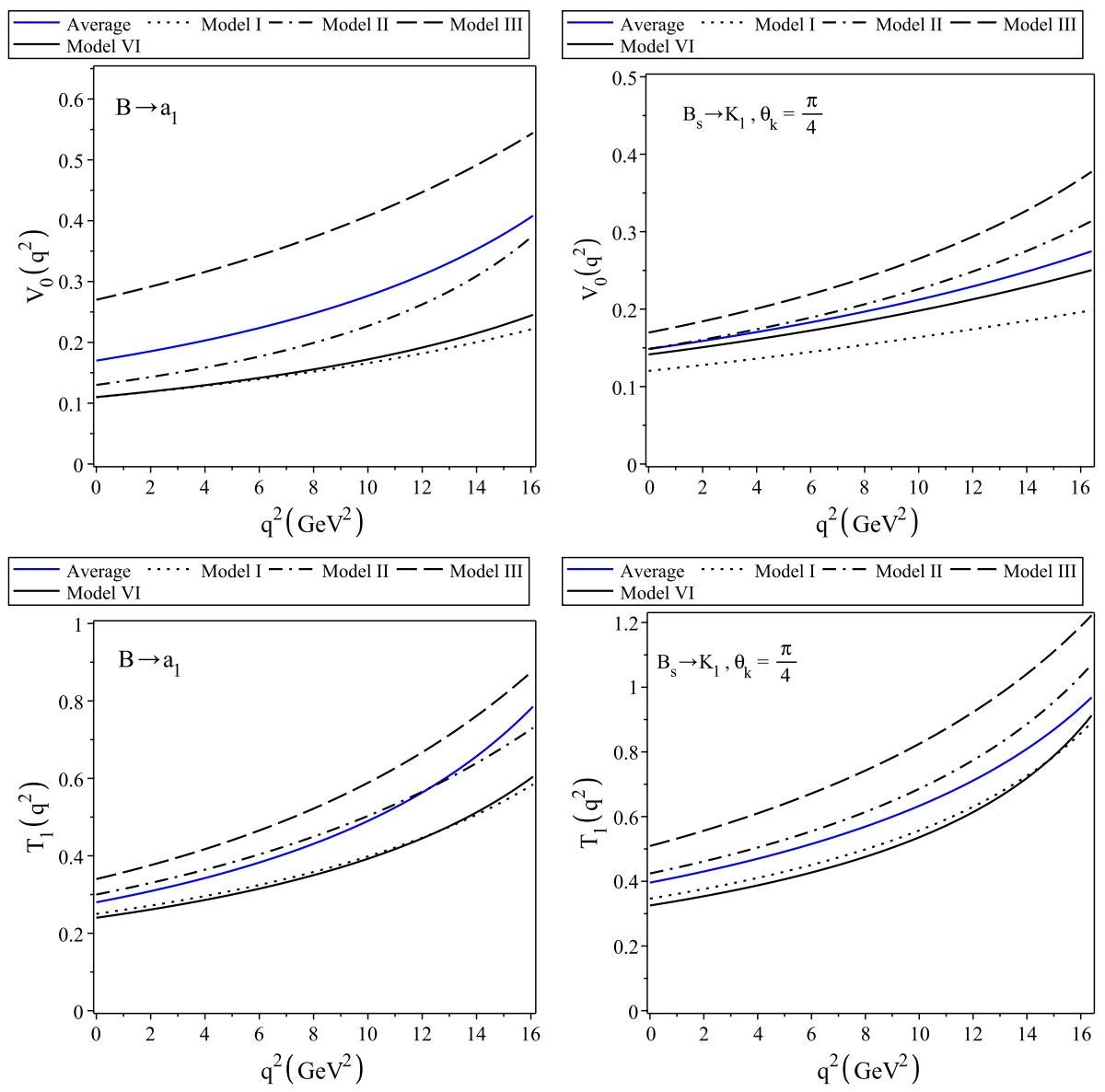

FIG. 3: The form factors $V_{0}$ and $T_{1}$ of $B_{(s)} \rightarrow a_{1}\left(K_{1}\right)$ on $q^{2}$ with the four models (black color). Blue lines show the average form factors. 

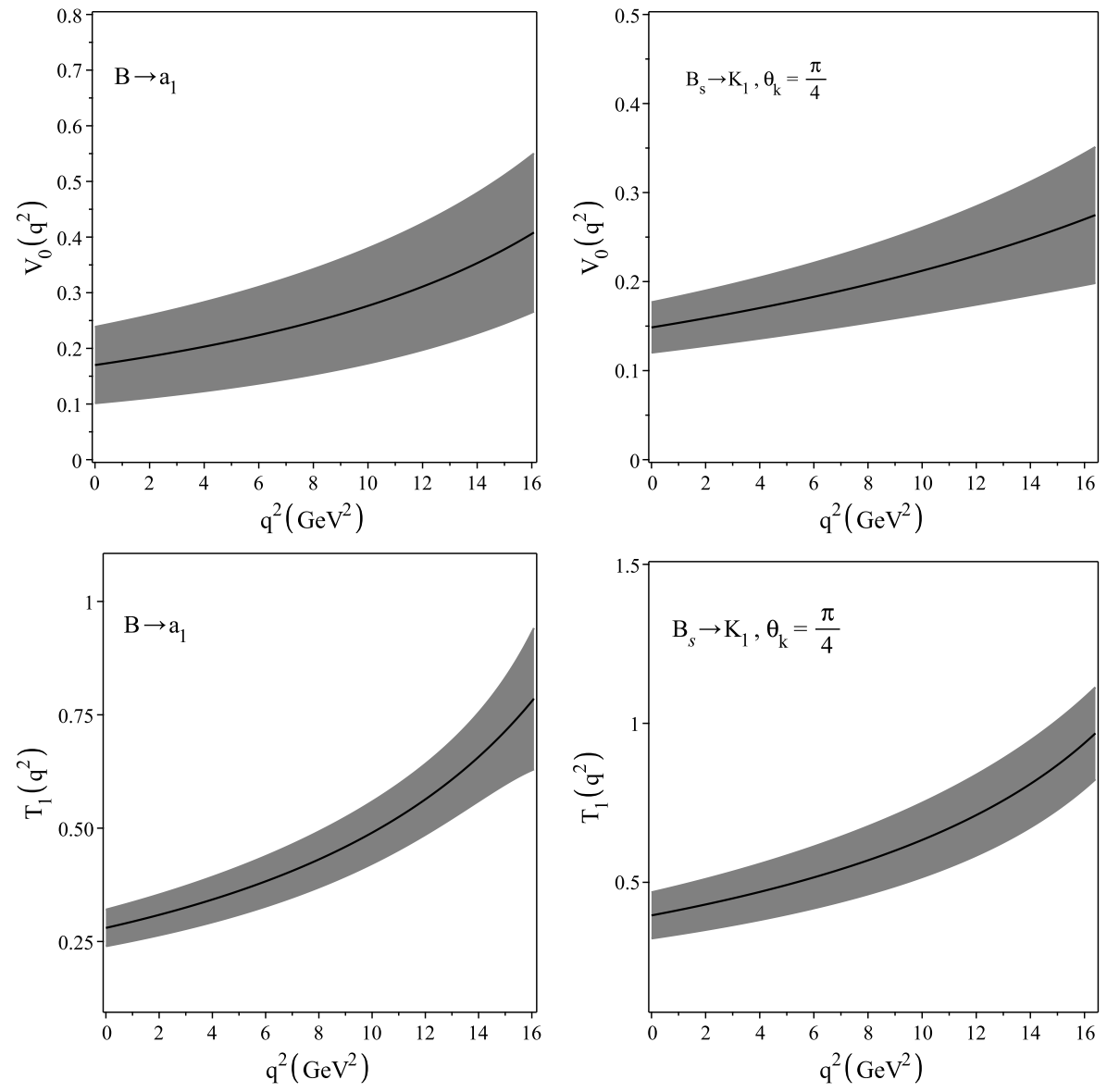

FIG. 4: The average form factors $V_{0}$ and $T_{1}$ of $B_{(s)} \rightarrow a_{1}\left(K_{1}\right)$ decays with their uncertainty regions.

TABLE IX: Central values of the Wilson coefficients used in the numerical calculations.

\begin{tabular}{ccccccccr}
\hline \hline$C_{1}$ & $C_{2}$ & $C_{3}$ & $C_{4}$ & $C_{5}$ & $C_{6}$ & $C_{7}^{\text {eff }}$ & $C_{9}$ & $C_{10}$ \\
\hline-0.248 & 1.107 & 0.011 & -0.026 & 0.007 & -0.031 & -0.313 & 4.344 & -4.669 \\
\hline \hline
\end{tabular}

TABLE X: Branching ratio values of the semileptonic $B_{(s)} \rightarrow a_{1}\left(K_{1}\right) \ell^{+} \ell^{-}$decays without and with LD effects using the form factors in the four models as well as the average form factors (AFF).

\begin{tabular}{cccccc}
\hline \hline Only SD effects & model-I & model-II & model-III & model-VI & AFF \\
\hline $\mathrm{BR}\left(B \rightarrow a_{1} \mu^{+} \mu^{-}\right) \times 10^{8}$ & $2.46 \pm 0.54$ & $2.77 \pm 0.58$ & $2.96 \pm 0.62$ & $2.43 \pm 0.50$ & $2.82 \pm 0.62$ \\
$\mathrm{BR}\left(B \rightarrow K_{1} \mu^{+} \mu^{-}\right) \times 10^{8}$ & $3.12 \pm 0.58$ & $3.41 \pm 0.82$ & $3.64 \pm 0.94$ & $3.09 \pm 0.57$ & $3.45 \pm 0.90$ \\
$\mathrm{BR}\left(B \rightarrow a_{1} \tau^{+} \tau^{-}\right) \times 10^{9}$ & $0.23 \pm 0.05$ & $0.25 \pm 0.05$ & $0.29 \pm 0.06$ & $0.22 \pm 0.04$ & $0.27 \pm 0.06$ \\
$\mathrm{BR}\left(B \rightarrow K_{1} \tau^{+} \tau^{-}\right) \times 10^{9}$ & $0.40 \pm 0.09$ & $0.44 \pm 0.10$ & $0.49 \pm 0.11$ & $0.39 \pm 0.08$ & $0.43 \pm 0.10$ \\
\hline $\mathrm{SD}+\mathrm{LD}$ effects & model-I & model-II & model-III & model-VI & AFF \\
\hline $\mathrm{BR}\left(B \rightarrow a_{1} \mu^{+} \mu^{-}\right) \times 10^{8}$ & $2.82 \pm 0.65$ & $3.18 \pm 0.74$ & $3.40 \pm 0.81$ & $2.79 \pm 0.64$ & $3.26 \pm 0.81$ \\
$\mathrm{BR}\left(B \rightarrow K_{1} \mu^{+} \mu^{-}\right) \times 10^{8}$ & $3.80 \pm 0.83$ & $4.16 \pm 0.91$ & $4.44 \pm 0.97$ & $3.76 \pm 0.82$ & $4.24 \pm 0.95$ \\
$\mathrm{BR}\left(B \rightarrow a_{1} \tau^{+} \tau^{-}\right) \times 10^{9}$ & $0.24 \pm 0.05$ & $0.26 \pm 0.05$ & $0.31 \pm 0.06$ & $0.23 \pm 0.04$ & $0.29 \pm 0.06$ \\
$\mathrm{BR}\left(B \rightarrow K_{1} \tau^{+} \tau^{-}\right) \times 10^{9}$ & $0.45 \pm 0.10$ & $0.50 \pm 0.11$ & $0.54 \pm 0.12$ & $0.44 \pm 0.09$ & $0.49 \pm 0.11$ \\
\hline \hline
\end{tabular}



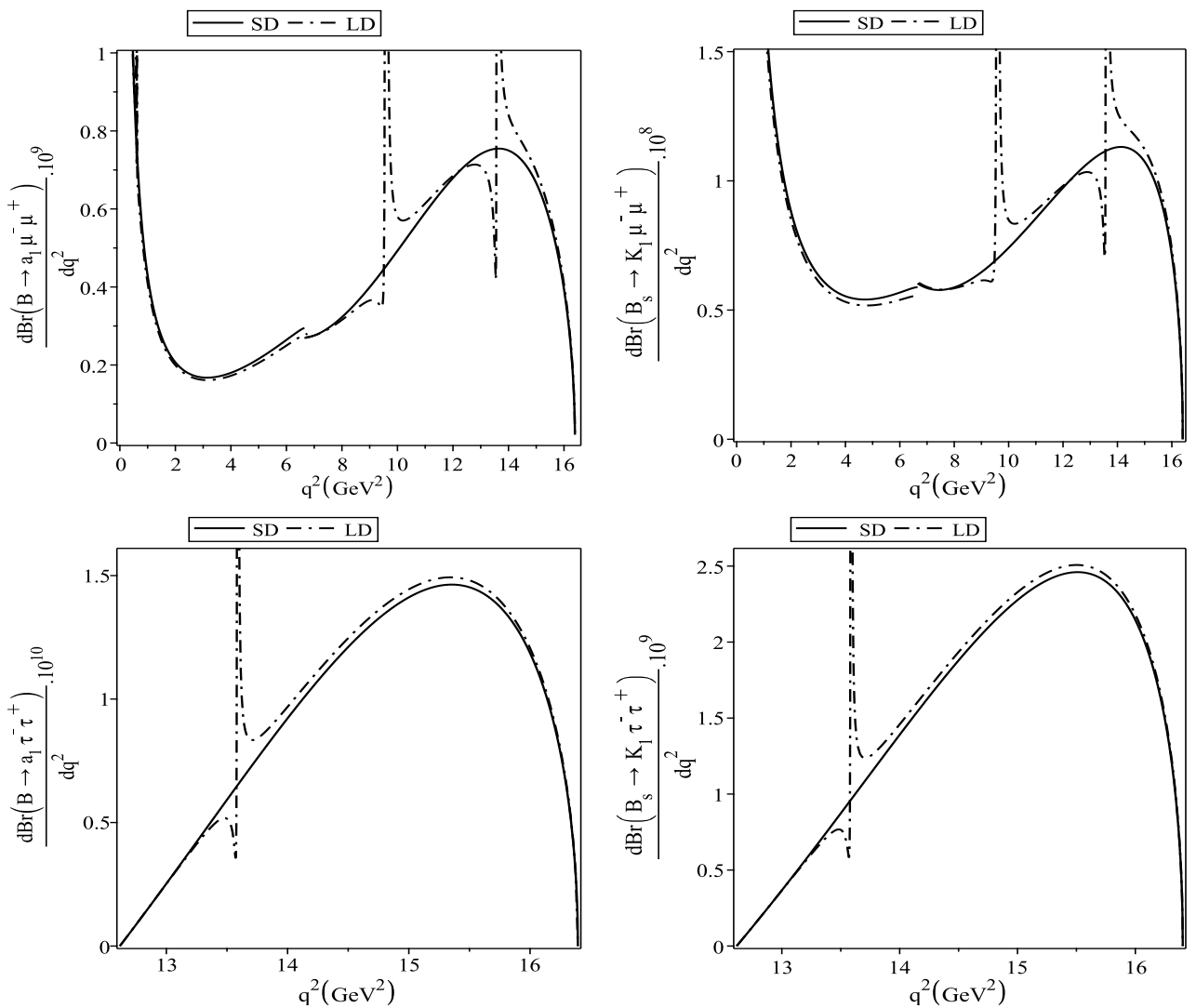

FIG. 5: The differential branching ratios of the semileptonic $B \rightarrow a_{1}\left(K_{1}\right) \ell^{+} \ell^{-}$for $\ell=\mu, \tau$ decays on $q^{2}$ with and without LD effects using the average form factors. 


\section{Appendix}

In this appendix, the explicit expressions for the form factors of $B \rightarrow a_{1} \ell^{+} \ell^{-}$decays are given.

$$
\begin{aligned}
& V_{2}\left(q^{2}\right)=\frac{f_{B} m_{B}\left(m_{B}-m_{a_{1}}\right)}{f_{a} m_{a_{1}}} e^{\frac{m_{a_{1}}^{2}}{M^{2}}}\left\{\int_{0}^{\sigma_{0}} d \sigma\left[\frac{\sigma}{2 \bar{\sigma}} \varphi_{+}\left(\omega^{\prime}\right) e^{-\frac{s}{M^{2}}}+\frac{1+\sigma}{2 \bar{\sigma}}\left(\widetilde{\varphi}_{+}\left(\omega^{\prime}\right)-\widetilde{\varphi}_{-}\left(\omega^{\prime}\right)\right) e^{-\frac{s}{M^{2}}}\right]\right. \\
& +\hat{\mathcal{L}}\left[\frac{(\sigma-2)(2 u-1)}{2 \bar{\sigma}^{2} M^{2}}\left(\Psi_{A}-\Psi_{V}\right) e^{-\frac{s}{M^{2}}}-\frac{(\sigma-2)(u-1)}{\bar{\sigma}^{2} M^{2}} \Psi_{V} e^{-\frac{s}{M^{2}}}+\frac{6 u-1}{2 \bar{\sigma}^{2} M^{2}} \widetilde{X}_{A} e^{-\frac{s}{M^{2}}}\right. \\
& \left.\left.-\frac{\sigma(2 u+1)-2}{2 \bar{\sigma}^{2} M^{2}} \widetilde{X}_{A} \frac{d}{d \sigma} e^{-\frac{s}{M^{2}}}-\frac{m_{B}^{2}(1+\sigma)(1-2 u)}{\bar{\sigma}^{2} M^{4}} \widetilde{Y}_{A} e^{-\frac{s}{M^{2}}}\right]\right\}, \\
& V_{0}\left(q^{2}\right)-V_{3}\left(q^{2}\right)=\frac{f_{B} m_{B} q^{2}}{f_{a} m_{a_{1}}^{2}} e^{\frac{m_{a_{1}}^{2}}{M^{2}}}\left\{\int_{0}^{\sigma_{0}} d \sigma\left[\frac{\sigma}{\bar{\sigma}} \varphi_{+}\left(\omega^{\prime}\right) e^{-\frac{s}{M^{2}}}+\frac{\sigma^{2}}{\bar{\sigma}^{2}}\left(\widetilde{\varphi}_{+}\left(\omega^{\prime}\right)-\widetilde{\varphi}_{-}\left(\omega^{\prime}\right)\right) e^{-\frac{s}{M^{2}}}\right]\right. \\
& +\hat{\mathcal{L}}\left[\frac{\sigma(1-2 u)}{\bar{\sigma}^{2} M^{2}}\left(\Psi_{A}-\Psi_{V}\right) e^{-\frac{s}{M^{2}}}+\frac{2 \sigma(1-u)}{\bar{\sigma}^{2} M^{2}} \Psi_{V} e^{-\frac{s}{M^{2}}}-\frac{4 u-2}{\bar{\sigma}^{2} M^{2}} \widetilde{X}_{A} e^{-\frac{s}{M^{2}}}\right. \\
& \left.\left.+\frac{\sigma(1-2 u)}{\bar{\sigma}^{2} M^{2}} \tilde{X}_{A} \frac{d}{d \sigma} e^{-\frac{s}{M^{2}}}-\frac{\sigma^{2} m_{B}^{2}(2-4 u)}{\bar{\sigma}^{3} M^{4}} \widetilde{Y}_{A} e^{-\frac{s}{M^{2}}}\right]\right\} \\
& A\left(q^{2}\right)=\frac{2 f_{B} m_{B}}{f_{a} m_{a_{1}}\left(m_{B}-m_{a_{1}}\right)} e^{\frac{m_{a_{1}}^{2}}{M^{2}}}\left\{\int_{0}^{\sigma_{0}} d \sigma\left[\frac{\sigma}{\bar{\sigma}} \varphi_{+}\left(\omega^{\prime}\right) e^{-\frac{s}{M^{2}}}-\frac{\sigma}{\bar{\sigma}}\left(\widetilde{\varphi}_{+}\left(\omega^{\prime}\right)-\widetilde{\varphi}_{-}\left(\omega^{\prime}\right)\right) e^{-\frac{s}{M^{2}}}\right]\right. \\
& \left.-\hat{\mathcal{L}}\left[\frac{\sigma(1-2 u)}{\bar{\sigma}^{2} M^{2}}\left(\Psi_{A}-\Psi_{V}\right) e^{-\frac{s}{M^{2}}}-\frac{2 \sigma(1-u)}{\bar{\sigma}^{2} M^{2}} \Psi_{V} e^{-\frac{s}{M^{2}}}-\frac{2 \sigma m_{B}^{2}}{\bar{\sigma}^{2} M^{4}}\left(\widetilde{X}_{A}-\widetilde{Y}_{A}\right) e^{-\frac{s}{M^{2}}}\right]\right\}, \\
& T_{1}\left(q^{2}\right)=\frac{f_{B} m_{B}}{2 m_{a_{1}} f_{a_{1}}} e^{\frac{m_{a_{1}}^{2}}{M^{2}}}\left\{\int _ { 0 } ^ { \sigma _ { 0 } } d \sigma \left[\frac{\sigma}{\bar{\sigma}} \varphi_{+}\left(\omega^{\prime}\right) e^{-\frac{s}{M^{2}}}+\frac{1}{M^{2}}\left(\widetilde{\varphi}_{+}\left(\omega^{\prime}\right)-\widetilde{\varphi}_{-}\left(\omega^{\prime}\right)\right) \frac{d}{d \sigma} e^{-\frac{s}{M^{2}}}\right.\right. \\
& \left.+\frac{\mathcal{M}^{2}\left(\bar{\sigma}+\sigma^{2}\right)+\bar{\sigma}+1}{\bar{\sigma}^{2} M^{2}}\left(\widetilde{\varphi}_{+}\left(\omega^{\prime}\right)-\widetilde{\varphi}_{-}\left(\omega^{\prime}\right)\right) e^{-\frac{s}{M^{2}}}\right]+\hat{\mathcal{L}}\left[\frac{\sigma^{2}(1-u)}{2 \bar{\sigma}^{2} M^{2}} \Psi_{V} e^{-\frac{s}{M^{2}}}\right. \\
& +\frac{\sigma^{2}(1-2 u)}{\bar{\sigma}^{2} M^{2}}\left(\Psi_{A}-\Psi_{V}\right) e^{-\frac{s}{M^{2}}}+\frac{\mathcal{M}^{2}\left(\bar{\sigma}^{2}(\sigma-1)+2 \bar{\sigma}-\sigma\right)}{\bar{\sigma} M^{2}}\left(\widetilde{X}_{A}-\widetilde{Y}_{A}\right) e^{-\frac{s}{M^{2}}} \\
& -\left(\frac{3 \bar{\sigma}^{2}-2 \sigma^{2}}{\bar{\sigma}^{2}}+\frac{2 \mathcal{M}^{2} u}{M^{4}}-\frac{2(4 \bar{\sigma}+3) u}{4 \bar{\sigma} M^{2}}\right)\left(\widetilde{X}_{A}-\widetilde{Y}_{A}\right) e^{-\frac{s}{M^{2}}}-\frac{\mathcal{M}^{2} u}{M^{4}} \widetilde{X}_{A} e^{-\frac{s}{M^{2}}} \\
& \left.\left.+\frac{(2 \bar{\sigma}+3) u}{2 \bar{\sigma} M^{2}} \widetilde{X}_{A} e^{-\frac{s}{M^{2}}}\right]\right\} \\
& T_{2}\left(q^{2}\right)=\frac{f_{B} m_{B}}{2 f_{a} m_{a_{1}}\left(m_{B}^{2}-m_{a_{1}}^{2}\right)} e^{\frac{m_{a_{1}}^{2}}{M^{2}}}\left\{\int _ { 0 } ^ { \sigma _ { 0 } } d \sigma \left[\left(s+\sigma m_{B}^{2}\right) \varphi_{+}\left(\omega^{\prime}\right) e^{-\frac{s}{M^{2}}}+\left(\frac{\mathcal{M}^{\prime 2}}{2}+\frac{\mathcal{M}^{4}}{M^{2}}\right)\right.\right. \\
& \left.\times\left(\widetilde{\varphi}_{+}\left(\omega^{\prime}\right)-\widetilde{\varphi}_{-}\left(\omega^{\prime}\right)\right) e^{-\frac{s}{M^{2}}}\right]+\hat{\mathcal{L}}\left[(1-2 u) \Psi_{A} \frac{d}{d \sigma} e^{-\frac{s}{M^{2}}}+(4 u-3) \Psi_{V} \frac{d}{d \sigma} e^{-\frac{s}{M^{2}}}\right. \\
& \left.\left.+(4 u-2)\left(\frac{3}{\bar{\sigma}^{2}}+\frac{\mathcal{M}^{\prime} 2}{\bar{\sigma}^{2} M^{2}}-\frac{\mathcal{M}^{4}}{2 \bar{\sigma}^{2} M^{4}}\right)\left(\widetilde{X}_{A}-\widetilde{Y}_{A}\right) e^{-\frac{s}{M^{2}}}+(4 u-2) \frac{\mathcal{M}^{2}}{\bar{\sigma}^{2} M^{2}} \widetilde{Y}_{A} e^{-\frac{s}{M^{2}}}\right]\right\}, \\
& T_{3}\left(q^{2}\right)=\frac{f_{B} m_{B}}{f_{a} m_{a_{1}}} e^{\frac{m_{a_{1}}^{2}}{M^{2}}}\left\{\int _ { 0 } ^ { \sigma _ { 0 } } d \sigma \left[\frac{\sigma}{\bar{\sigma}} \varphi_{+}\left(\omega^{\prime}\right) e^{-\frac{s}{M^{2}}}-\left(\frac{4}{M^{2}}-\frac{4 \sigma \mathcal{M}^{2}}{\bar{\sigma} M^{4}}-\frac{2 \sigma^{2}\left(\mathcal{M}^{2}+\mathcal{M}^{\prime 2}\right)}{\bar{\sigma} M^{4}}\right)\right.\right. \\
& \left.\times\left(\widetilde{\varphi}_{+}\left(\omega^{\prime}\right)-\widetilde{\varphi}_{-}\left(\omega^{\prime}\right)\right) \frac{e^{-\frac{s}{M^{2}}}}{\bar{\sigma}}\right]+\hat{\mathcal{L}}\left[(1-2 u)\left(\frac{1}{M^{2}}+\frac{\mathcal{M}^{2}}{M^{4}}-\frac{m_{B}^{2}}{M^{4}}\right)\left(\Psi_{A}-\Psi_{V}\right) e^{-\frac{s}{M^{2}}}\right. \\
& \left.\left.+\frac{\sigma(u-1)}{\bar{\sigma}^{2} M^{2}} \Psi_{V} e^{-\frac{s}{M^{2}}}+(1-2 u)\left(\frac{1}{\bar{\sigma}^{2} M^{2}}+\frac{2 \sigma}{\bar{\sigma}^{3} M^{2}}-\frac{\sigma \mathcal{M}^{2}}{\bar{\sigma}^{3} M^{4}}\right)\left(\widetilde{X}_{A}-2 \widetilde{Y}_{A}\right) e^{-\frac{s}{M^{2}}}\right]\right\},
\end{aligned}
$$


where:

$$
\hat{\mathcal{L}} \equiv \int_{0}^{\sigma_{0}} d \sigma \int_{0}^{\omega^{\prime}} d \omega \int_{\omega^{\prime}-\omega}^{\infty} \frac{d \xi}{\xi}
$$

$\omega^{\prime}=\sigma m_{B}$ and $\widetilde{m}_{B}^{2}=m_{B}^{2}(1+\sigma)-q^{2} / \bar{\sigma}$, also:

$$
\begin{array}{ll}
s=\sigma m_{B}^{2}-\frac{\sigma}{\bar{\sigma}} q^{2}, & \bar{\sigma}=1-\sigma, \\
\widetilde{\phi}_{ \pm}=\int_{0}^{\omega} d \tau \phi_{ \pm}(\tau), & \widetilde{X}_{A}=\int_{0}^{\omega} d \tau X_{A}(\tau, \xi), \\
\mathcal{M}^{2}=\widetilde{m}_{B}-2 \sigma m_{B}^{2}, & \mathcal{M}^{4}=\widetilde{m}_{B}^{4}-4 s m_{B}^{2}, \\
\sigma_{0}=\frac{s_{0}+m_{B}^{2}-q^{2}-\sqrt{\left(s_{0}+m_{B}^{2}-q^{2}\right)^{2}-4 s_{0} m_{B}^{2}}}{2 m_{B}^{2}} .
\end{array}
$$

$$
\begin{aligned}
& u=\frac{\omega^{\prime}-\omega}{\xi}, \\
& \widetilde{Y}_{A}=\int_{0}^{\omega} d \tau Y_{A}(\tau, \xi), \\
& \mathcal{M}^{\prime 2}=2 m_{B}^{2}-\widetilde{m}_{B}^{2},
\end{aligned}
$$

[1] I. I. Balitsky, V. M. Braun and A. V. Kolesnichenko, Nucl. Phys. B 312, 509 (1989).

[2] V. M. Braun and I. E. Halperin, Z. Phys. C 44, 157 (1989).

[3] V. L. Chernyak and I. R. Zhitnitsky, Nucl. Phys. B 345, 137 (1990).

[4] V. M. Belyaev, A. Khodjamirian and R. Ruckl, Z. Phys. C 60, 349 (1993).

[5] A. Ali, V. M. Braun and H. Simma, Z. Phys. C 63, 437 (1994).

[6] V. M. Belyaev, V. M. Braun, A. Khodjamirian and R. Ruckl, Phys. Rev. D 51, 6177 (1995).

[7] A. Khodjamirian, R. Ruckl, S. Weinzierl and O. I. Yakovlev, Phys. Lett. B 410, 275 (1997).

[8] P. Ball and V. M. Braun, Phys. Rev. D 58, 094016 (1998).

[9] E. Bagan, P. Ball and V. M. Braun, Phys. Lett. B 417, 154 (1998).

[10] P. Ball, JHEP 9809, 005 (1998).

[11] P. Ball and R. Zwicky, JHEP 0110, 019 (2001).

[12] P. Ball and R. Zwicky, Phys. Rev. D 71, 014015 (2005).

[13] A. Szczepaniak, E. M. Henley and S. J. Brodsky, Phys. Lett. B 243, 287 (1990).

[14] A. G. Grozin and M. Neubert, Phys. Rev. D 55, 272 (1997).

[15] M. Beneke and T. Feldmann, Nucl. Phys. B 592, 3 (2001).

[16] P. Ball and E. Kou, JHEP 0304, 029 (2003).

[17] A. Khodjamirian, T. Mannel and N. Offen, Phys. Lett. B 620, 52 (2005).

[18] A. Khodjamirian, T. Mannel and N. Offen, Phys. Rev. D 75, 054013 (2007).

[19] H. Hatanaka and K. C. Yang, Phys. Rev. D 78, 074007 (2008).

[20] L. Burakovsky and T. Goldman, Phys. Rev. D 57, 2879 (1998).

[21] M. Suzuki, Phys. Rev. D 47, (1993) 1252.

[22] A. J. Buras and M. Muenz, Phys. Rev. D 52, 186 (1995).

[23] T. M. Aliev, V. Bashiry and M. Savci, Phys, Rev, D 72, 034031 (2005).

[24] M. Jezabek and J. H. Kuhn, Nucl. Phys. B 320, 20 (1989).

[25] M. Misiak, Nucl. Phys. B 439, 461 (1995).

[26] P. Colangelo, F. De Fazio, P. Santorelli and E. Scrimieri, Phys. Rev. D 53, 3672 (1996).

[27] Z. G.Wang ,Phys, Lett. B 666, 477-482 (2008).

[28] V. M. Braun, D. Yu. Ivanov and G. P. Korchemsky, Phys. Rev. D 69, 034014 (2004).

[29] F. De Fazio, T. Feldmann and T. Hurth, JHEP 0802, 031 (2008).

[30] Y. M. Wang and Y. L. Shen, Nucl. Phys. B 898, 563 (2015).

[31] Y. Li, J. Hua and K. Yang, Eur. Phys. J. C 71, 1775 (2011).

[32] K. A. Olive et al., Particle Data Group, Chin. Phys. C 38, 090001 (2014).

[33] K. C. Yang, Nucl. Phys. B 776, 187 (2007).

[34] Z. G. Wang, W. M. Yang and S. L. Wan, Nucl. Phys. A 744, 156 (2004).

[35] R. Li, C. Lu and W. Wang, Phys. Rev. D 79, 034014 (2009).

[36] S. Momeni, R. Khosravi, and F. Falahati, Phys. Rev. D 95, 016009 (2017).

[37] R. Khosravi, Eur. Phys. J. C 75, 220 (2015).

[38] C. Q. Geng and C. C. Liu, J. Phys. G 29, 1103 (2003).

[39] A. Ali, P. Ball, L. T. Handoko and G. Hiller, Phys. Rev. D 61074024 (2000). 